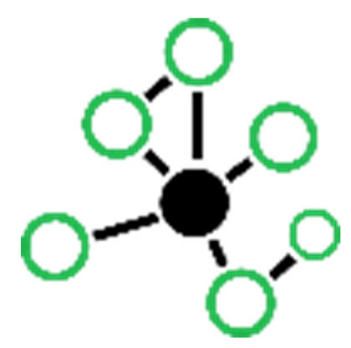

\title{
Farm Data Train
}

\author{
Finkers, R. ${ }^{1}$, \\ ${ }^{1}$ Wageningen University \& Research, Droevendaalsesteeg 1, 6708PB Wageningen, The Netherlands \\ Corresponding author's e-mail: richard.finkers@wur.nl
}

The Farm Data Train's main goal is connecting agricultural data to make them more useable. Modern farm management, for example, requires ever increasing amounts of data. But today, this information can not easily be used because the data are produced and managed by various manufacturers of machines, authorities, and more and more by farmers themselves.

These stakeholders collect and manage their data in different ways, making the data hard to find and use. Furthermore, as some of it is public and others need to remain private, specific farm information is often very business critical. As a consequence, farm data cannot be used easily by farmers themselves, nor by agronomists and researchers.

The Farm Data Train goes to the root of this problem by building FAIR Data Stations. FAIR protocols ensure that data are Findable, Accessible, Interoperable and Reusable. 